\title{
Der Eigensinn des Körpers
}

\author{
Carla van Kaldenkerken
}

Eingegangen: 20. Oktober 2020 / Angenommen: 24. November 2020 / Online publiziert: 26. Januar 2021 (C) Springer Fachmedien Wiesbaden GmbH, ein Teil von Springer Nature 2021

Zusammenfassung Embodiment (Verkörperung) ist als wissenschaftlicher Begriff für interdisziplinäre Diskurse eingeführt, die die enge, wechselseitige Verbundenheit von Körper, Geist und Umwelt untersuchen und eine systematische Integration der Kategorie „Körper“ in ihre Konzeptionen verfolgen. Ist es sinnvoll und lässt sich das überwiegend unbewusst ablaufende leibliche Geschehen absichtsvoll in die Beratung einbinden? Es wird für eine achtsame, den Eigensinn des Körpers respektierende und kenntnisreiche Übertragung des Konzepts in die Beratung plädiert. Bei einer konsequenten Beachtung der bidirektionalen Verbundenheit mit der Umwelt hat das Konzept auch eine politische Dimension.

Schlüsselwörter Embodiment · Gesellschaftliche Vorannahmen · Zyklische Zeit

\section{The self-will of the body}

Abstract Embodiment (Verkörperung) has been introduced as a scientific term for interdisciplinary discourses that investigate the close, reciprocal connection between body, mind and environment and pursue a systematic integration of the category "body" in their conceptions. Does it make sense and can the predominantly unconsciously occurring bodily events be intentionally integrated into counselling and coaching? The paper argues for a mindful, knowledgeable transfer of the concept into counselling and coaching that respects the body's inherent qualities (Eigensinn). If the bi-directional connection with the environment is consistently taken into account, the concept also has a political dimension.

Keywords Embodiment $\cdot$ Social assumptions $\cdot$ Cyclical time

C. van Kaldenkerken $(\square)$

Nienbergen 13, 29468 Bergen, Deutschland

E-Mail: vankaldenkerken@step-beratung.de 


\section{Vorbemerkung}

Die Einschränkungen in der Beratungspraxis durch die Verhaltensmaßnahmen zur Eindämmung der Coronavirus-Pandemie geben uns unübersehbare Hinweise zur Rolle des Körpers in der Beratung. Das nonverbale, meist un- und randbewusste leibliche Geschehen, die latente Beteiligung, Rolle und Mitwirkung im Beratungsprozess werden durch den Verlust von Nähe, Beweglichkeit, sichtbarer Mimik und Gestik so eindrücklich deutlich und werfen Fragen wie diese auf:

1. Gibt es die zwischenleiblichen Synchronieprozesse auch in einer Video-Konferenz?

2. Was verändert sich im Kontakt, wenn wir wegen der Mund-Nasenmaske die Mimik nicht erkennen können?

3. Welche Veränderungen in der Beratung erleben wir durch das Fehlen des Gesamteindrucks von Körper, Bewegung, Mimik, Sprache usw., wenn wir in der Videoberatung nur das Gesicht sehen und die Stimme verzerrt hören?

Auch wenn in Beratungen der Fokus überwiegend auf die explizite, verbale Interaktion ausgerichtet ist, spielt die präverbale Beteiligung und die zwischenleibliche Interaktion und Resonanz eine zentrale Rolle. Die Einschränkungen in der Gestaltung von Nähe und Distanz im sozialen Miteinander, das Fehlen an Orientierung durch Mimik, Gestik, Stimmmodulation und anderen nonverbalen Ausdrucksformen wird häufig als großer Verlust beschrieben. Erst mit einer „Störung“, hier mit dem Fehlen einiger Aspekte in der unbewussten, nonverbalen Verbindung, tritt die latente Beteiligung des Körpers in die explizite Aufmerksamkeit und in das Bewusstsein. „Es bedarf der körperlichen Gesten, des Mienenspiels, der Momente des Schweigens, der Körpersprache und sogar des Geruchs, der zitternden Hände, des Errötens und des Schwitzens, denn all dies spricht und gehört zur menschlichen Kommunikation“, plädiert auch Papst Franziskus (2020) für den Stellenwert der „Leiblichkeit“ in der Sozialenzyklika „Fratelli tutti““.

Zur Beantwortung der oben genannten Fragen hat das Embodimentkonzept (im Folgenden „Verkörperung“ genannt) eine Fülle an Anregungen zu bieten. Die Beteiligung des Körpers im gesamten Geschehen von Denken, Fühlen und Handeln, insbesondere das zwischenleibliche Resonanzgeschehen in der dyadischen Interaktion ist empirisch gut nachgewiesen (vgl. Tschacher und Bannwart, in diesem Heft).

Die Verkörperungsdiskurse in den Geistes-, Kognitions- und Sozialwissenschaften schließen philosophische Reflexionen zu der grundlegenden Fragestellung vom Verhältnis von Geist und Körper, die sich durch die gesamte Geschichte der Philosophie ziehen, mit ein. Die lange Tradition der Körperwahrnehmungs- und Achtsamkeitspraxis, einige methodische Schulen, die psychosomatische Medizin und Körperpsychotherapie haben wichtige Grundlagen gelegt und bereits ein großes Repertoire an Interventionsmöglichkeiten entwickelt. Die Verkörperungsperspektive stellt den tief verwurzelten cartesianischen Körper-Geist-Dualismus und damit auch ein rein kognitives Vorgehen in der Beratung radikal infrage. Konsequent verfolgt, beschränkt sich das Verkörperungskonzept nicht auf methodische Überlegungen, sondern versteht sich als ein durchgängiges Prinzip, eine zusätzliche Perspektive und Einstellung. 
Nach einem kurzen Überblick möchte ich ausgewählte Aspekte vertiefen und an drei Leitgedanken orientieren:

1. Wenn die cartesianische Trennung nicht nur eine Betrachtungsweise, sondern auch ein gelebter Umgang mit sich selbst ist, stellt sich die Frage, in welcher Weise Berater/innen sich die Verkörperungsperspektive erschließen können.

2. Der Einschätzung der Verkörperungsthesen als Paradigmenwechsel und Erweiterung zu einem ganzheitlicheren Beratungsverständnis stehen kritische Bewertungen gegenüber, die die Konzepte als Neuaufguss bereits bekannter methodischer Traditionen, als neue Modewelle, als Verdinglichung und Selbstentfremdung durch eine Neurobiologisierung beschreiben und damit wichtige Einwände zur Sprache bringen, die als Fragestellungen mitlaufen.

3. Die grundlegende Perspektive einer unauflösbaren Verbindung von Körper, Geist und Umwelt schließt gesellschaftliche Themen und Dynamiken wie die Folgen der Beschleunigung, Überforderung und die Bedrohung unserer Lebensgrundlagen nicht aus. Der Frage, in welcher Weise diese Dimension in Beratungen relevant wird, bildet den Abschluss des Beitrages.

\section{Eine kurze Einführung}

Arbeitsweltliche Berater/innen müssen sich angesichts der vielschichtigen Aspekte ihrer Formate mit Theorien zu Person, Gruppe, Organisation und immer wieder mit neuen Themen im Zusammenhang mit den Veränderungen in der Arbeitswelt beschäftigen. Die leibliche Basis all unserer Lebensvollzüge und der spezifischen menschlichen Produktivität, das Erleben von Vitalität, Selbstwirksamkeit und Sinnhaftigkeit, Verbundenheit und Zufriedenheit ist mit Antonovsky (1997) bereits Grundwissen von Berater/innen und erfährt mit der Verkörperungsperspektive neue Relevanz. Die Beschäftigung mit dem Körper beschränkt sich allerdings häufig auf Themen der Verunsicherung und Überforderung, der Gesunderhaltung in dysfunktionalen Verhältnissen, als Teil von Rollenberatung und im Zusammenhang mit methodischen Überlegungen.

Bei dem Versuch, sich zu Angeboten, Publikationen und Weiterbildungen zum Thema Embodiment und verkörperte Beratung zu informieren, schwirrt einem schnell der Kopf. Unübersichtlich, konzeptionell unterschiedlich, wenn nicht sogar widersprüchlich präsentieren sich unter diesem „Label“ Weiterbildungs- und Beratungsangebote. Es scheint einige Missverständnisse zu geben, wenn Embodiment auf die wechselseitige Beeinflussung von Körper und Geist und zur Technik und Methode verkürzt wird. Irritationen entstehen außerdem, wenn mithilfe der ,Technik“ die Optimierung der Beratung oder sogar des Beratungskunden versprochen wird. Es kann auch nicht nur um die Übertragung körperpsychotherapeutischer und erlebniszentrierter Konzepte gehen, die sich zum Teil an ganz anderen Paradigmen orientieren, oder um eine einseitige Orientierung an Thesen neurowissenschaftlicher Forschung. Es drängt sich der Eindruck auf, dass die Kommerzialisierung des Themas die professionelle Verständigung zur Rolle der Verkörperungsperspektive in den arbeitsweltlichen Beratungsformaten bereits überholt hat. 
Demgegenüber ist in einem interdisziplinären, sich dynamisch entwickelnden Forschungsfeld in der Schnittstelle und gegenseitigen Anregung von Philosophie, Kognitionswissenschaft, Neurowissenschaften, Entwicklungspsychologie und Körperpsychotherapie ein spannender Verkörperungsdiskurs entstanden, der über die Traditionen und Paradigmen vieler körperpsychotherapeutischer Konzepte und erlebensorientierter Methoden hinausgeht. Vom ,body-turn“ in den Geistes-, Kultur- und Sozialwissenschaften ist die Rede. Wer sich mit der Verbindung von Körper/Leib, Geist und Kontext vertieft beschäftigt, kann nicht anders, als die „Zuständigkeit““ seines jeweiligen Fachgebietes zu verlassen und neugierig über den Tellerrand zu blicken. Und es wundert auch nicht, dass gesellschaftliche Themen, wie die Bedrohung unserer Natur und unserer eigenen Lebensgrundlage in solchen Konzeptionen konsequent mitbedacht werden (vgl. Petzold 2016).

Das grundlegende Verständnis, dass unsere Handlungen, Gedanken, Gefühle, Situationsbewertungen in fortlaufenden Wechselwirkungen (Bidirektionalität) mit physiologischen, motorischen und psychischen Prozessen und der Umwelt verbunden sind, stellt die tief verwurzelte Vorstellung vom Körper-Geist-Dualismus im wahrsten Sinne des Wortes vom Kopf auf die Füße und könnte auch in den arbeitsweltlichen Beratungsformaten eine grundlegende und kritische Überprüfung der Konzepte anregen. Vor dem Hintergrund der Verkörperungstheorien lässt sich der cartesianische Dualismus theoretisch schwer aufrechterhalten. Über methodische Überlegungen hinaus ist die Verkörperung als durchgängiges Prinzip und als Perspektive zu verstehen, die die grundlegende Leiblichkeit und Verkörperung aller menschlichen Erfahrungen berücksichtigt.

Das klingt einfacher, als es ist, wie ich noch ausführen werde, denn faktisch ist diese Vorstellung tief verankert. „Der Cartesianismus ist nicht bloß eine Theorie, sondern wir leben den Cartesianismus, und zwar nicht bloß in einer Betrachtungsweise, sondern im instrumentellen Umgang mit uns selbst“" (Böhme 2017, S. 52). Kommentierungen in Beratungen wie z. B.: ,er hat völlig kopflos reagiert, die Probleme sind ihr über den Kopf gewachsen, da haben wir uns lange den Kopf zerbrochen, oder das muss ich mir erstmal durch den Kopf gehen lassen“, zeugen von der Lokalisierung geistiger Vorgänge im Kopf. Die verärgerte Kommentierung, dass man sein Bauchgefühl missachtet habe, gibt wiederholt deutliche Hinweise, dass Körperempfindungen eine relevante Bedeutung bei Entscheidungsprozessen haben könnten, aber in der Arbeitsrealität häufig nicht haben.

Die berechtigte Sorge, dass eine größere Aufmerksamkeit für den Körper in der arbeitsweltlichen Beratung ein Übergriff auf das Private sei, ist nicht abzuweisen. Anders als in der Körperpsychotherapie verfolgen die verschiedenen Akteure und Auftraggeber/innen unterschiedliche Interessen. Kritische Betrachtungen warnen vor dem Übergriff auf den Körper als eine weitere Optimierungsreserve im Beschleunigungskarussell. Die verkörperte Sichtweise beleuchtet diesen Aspekt, weil die wechselseitigen Beeinflussungen mit organisationalen und gesellschaftlichen Variablen, wie z.B. die Veränderungen im Kontext von Digitalisierung und agilen Organisations- und Führungskonzepten, Teil der Perspektive ist. Bachmann (2020, S. 295) fragt, ob es etwas gibt, ,das nicht digitalisiert werden kann, das sich der Algorithmisierung entzieht, das so komplex, einzigartig und unberechenbar ist und sich daher nicht mit Maschinen herstellen lässt?“. 
Eine These wäre, dass sich eigenleibliche Rhythmen und die komplexe Selbstorganisation von Körper/Leib, Geist und Umwelt nicht immer in Passung mit den linearen Prozessen von Fortschritt und Wachstum bringen lassen und der Beschleunigungsdynamik Grenzen setzen.

\section{Erste Vertiefung: Die Körper-Leib-Unterscheidung}

Anders als im Englischen gibt es in der deutschen Sprache die Begriffe Körper und Leib. Die Unterscheidung ermöglicht die Beschreibung zweier Facetten, die untrennbar zusammengehören und das doppelseitige Verhältnis des Menschen zu seinem Körper deutlich machen. „Die Ausdrücke ,Leib“ und ,Körper' bilden ein sprachliches Kapital, das man nicht einfach verschleudern sollte, indem man von ,Körper spricht, wenn man den ,Leib“ meint“ (Waldenfels 2000, S. 15). „Der Mensch ist sein Körper, und er hat seinen Körper. Sein und Haben sind die zwei Weisen, in denen dem Mensch sein Körper gegeben ist. Körpersein und Körperhaben sind zwei Perspektiven auf die physische Existenz des Menschen, (...) zwei Facetten menschlichen Daseins, die untrennbar miteinander verbunden sind, sich wechselseitig bedingen, wobei ihr Verhältnis zueinander historisch-kulturell variabel ist" (Gugutzer 2015, S. 13).

Der Leib, der ich bin, ist der belebte, subjektiv gespürte Körper, den ich nicht verlassen kann. Jörg Sternagel (2012, S. 120) beschreibt den Leib als Handlungsund Beziehungsmitte, in dem sich Selbst- und Weltbezug verschränken. Die meisten unserer Lebensvollzüge vollziehen sich unbemerkt als latentes somatisches Hintergrunderleben (Damasio 2004, 2011). Leibsein ist unauffällig und versteht sich nicht von selbst. Der Leib liegt sozusagen im Rücken und lässt sich nicht in den Blick nehmen, sondern nur spürend erfahren und nur schwer erforschen und theoretisch vermitteln. Der Körper ist der materielle, sichtbare, von außen zu sehende, zu berührende, ,dinghafte“ Körper. Der Körper wird in der Perspektive der Fremderfahrung zum Gegenstand, zum Objekt, das man betrachten, behandeln, funktionalisieren und manipulieren kann, und im Extremfall zum sinn- und selbstwertsteigernden Objekt und Statussymbol, zum Körper, den man hat.

Waldenfels trifft die Unterscheidung zwischen dem Leib, der wir sind als Gesamtheit des Selbst, und dem Körper, den wir haben als Materialität des Leibes. Der Vorschlag von Gallagher (2012) sieht die Unterscheidung zwischen der unbewussten Dimension des Körperschemas und der bewussten Dimension des Körperbildes. Plessners (1982) Unterscheidung von Körper und Leib und seine Konzeption der exzentrischen Positionalität beschreibt diese Doppelheit von Leib (sein) und Körper (haben) als zwei prinzipielle „Ordnungen“ der Stellung in der Welt, positioniert in einem Zentrum-Außenwelt-Verhältnis. Mit der sprachlichen Unterscheidung zwischen dem Körper und dem Leib kommt die Fähigkeit des Menschen zum Ausdruck, sich von seinem Leib auch distanzieren zu können, ihn zum Gegenstand zu machen, sich zu dem subjektiv gespürten Leib ins Verhältnis zu setzen.

In der Innenperspektive bildet der Leib das Zentrum allen Erlebens und ist räumlich und zeitlich im Hier und Jetzt gebunden. Die exzentrische Position beschreibt die Fähigkeit des Menschen, zu diesem zentrischen Innenerleben eine Art Außenper- 
spektive auf sich selbst und die umgebende Welt einzunehmen und damit Abstand zum Erleben zu nehmen. Die Fähigkeit zu dieser Selbstdistanzierung nennt Plessner (1982) „Exzentrizität“. Diese exzentrische Position, eine dritte Perspektive durch den Blick der anderen, ist nicht nur eine reflexive Position. Durch den Blick der anderen werden auch kulturelle und soziale Orientierungsmaßstäbe vermittelt.

Obwohl der Leib und der Körper etwas grundsätzlich Verschiedenes sind, sind sie in einer Doppelrolle verbunden. Man kann sich diese Verbindung von Körper und Leib wie ein ständiges Oszillieren zwischen dem latenten Hintergrunderleben der Leiblichkeit und der bewussten Seite der Körperlichkeit vorstellen. „Exzentrizität ist also nicht einfach durch das Bestehen eines Doppelten von Körper-sein und Körper-haben charakterisiert" (Schürmann 2012, S. 218), sondern meint sowohl das Vermögen und die Möglichkeit des Menschen als auch die Aufgabe, sich in seinem Lebensvollzug zu dieser Doppelheit ins Verhältnis zu setzen. Bei Stress, Konflikten und Krisen sinkt die Fähigkeit, die exzentrische Position einzunehmen und zwischen den Perspektiven zu oszillieren. Die Fähigkeit zur Selbstbestimmung und zur Selbstvertretung z.B. in Konflikten, zur komplexen Reflexion und abgewogenen Entscheidungen geht zunehmend verloren.

Böhme attestiert der technischen Zivilisation ein hohes Maß an Selbstentfremdung und einen spezifischen, kulturellen Umgang mit dem Körper. „Denn wir leben in der technischen Zivilisation und sind durch die darin dominanten Lebensformen immer schon auf eine bestimmte Weise Leib zu sein einsozialisiert, bzw. einkultiviert und die sind derart, dass wir über unseren Leib in der Regel hinwegleben, ihn instrumentalisieren und ihn zum Körper objektivieren“ (Böhme 2017, S. 73).

Eine hilfreiche Orientierung bietet diese Konzeption auch für die Indikation und Auswahl methodischer Interventionen und Elementen (vgl. 5. Vertiefung) anhand der drei Perspektiven. Mit dem Rollentausch oder der Metaposition aus der Zentralität herauszutreten, in eine andere Perspektive einzutreten, zwischen den beiden zu wechseln und das Ganze und seine Wechselwirkungen von außen zu betrachten, sind methodische Elemente für die Erarbeitung einer komplexen Sichtweise. „Die Entwicklung von gesundem Eigensinn braucht auch psychologische Strategien, die geduldige Übung von wacher Wahrnehmung und einen weniger achtlosen Umgang mit uns selbst und anderen" (Milz 2019, S. 320). Eine verkörperte Selbstwahrnehmung schenkt den eigenen Wahrnehmungen, Empfindungen, Bewegungen eine interessierte, nicht bewertende Aufmerksamkeit. Somatics (somatische Praxis) wie z. B. die Feldenkrais- und Gindlermethode, Achtsamkeits- und Meditationsübungen, Tai Chi, Yoga, Naturerfahrungen u. a. sind Möglichkeiten, eine wache Wahrnehmung zu üben.

All diese Ansätze und Methoden können wieder zur Fremderfahrung werden, wenn sie in den Dienst der Beherrschung, Formung und Disziplinierung, Optimierung und Wertsteigerung eingesetzt werden. So bildet eine Körperwahrnehmungs-, Achtsamkeits- und Meditationspraxis nicht qua Zuwendung zum leiblichen Spüren schon leibliche Praxis. Die boomenden Achtsamkeits- und Meditationsangebote können im Kontext von Selbstmanagement auch in den Dienst des Funktionierens und der Optimierung gestellt werden, wie einige Autoren kritisch anmerken (Schindler 2020). 


\section{Zweite Vertiefung: Leibsein als Aufgabe}

„Wohl kaum eine Zeit hat der Wahrnehmung so wenig Vertrauen entgegengebracht wie die gegenwärtige“ (Fuchs 2020, S. 146). Leibsein als vertraut sein mit sich selbst versteht sich nicht mehr von selbst, so Böhme (s. oben), sondern wird zur Aufgabe. Sich selbst zu vertrauen, heißt, den eigenen Wahrnehmungen zu trauen, und wird nur über die Zuwendung zum leiblichen Erleben möglich. Auch die Selbstsicherheit ist gegründet im sicheren Kontakt und in vertiefter Kenntnis und Beziehung zu sich selbst. Der Leib ist Resonanzkörper, Bezugspunkt und Informationsquelle für die persönliche Orientierung, für die Bewertung von Situationen und Entscheidungen.

Supervision und Coaching sind keine Selbsterfahrungsformate. Es geht vordergründig nicht um die Entwicklung eigenleiblicher Spür-, Resonanz- und Schwingungsfähigkeit der Kunden, sondern mehr um die Bereitstellung eines Raums für Personen und Gruppen, in dem die Aufmerksamkeit auch für körperliche/leibliche Phänomene, Stimmungen und Emotionen möglich werden kann. Im Nachspüren leiblicher Eindrücke erhält man zusätzliche Auskünfte, die die verbalen, expliziten Informationen ergänzen, bestätigen oder auf Widersprüche hinweisen. Es geht nicht um den Vorrang emotionaler, somatischer Marker bei Entscheidungen und Bewertungen, sondern um zusätzliche Hinweise auf Stimmigkeit und Passung.

Für die Beratungspraxis wird im konkreten Gegenwartsbezug die verbal explizierte Situation oder Entscheidung auf eine zusätzliche Weise erkundet und vervollständigt. Wenn das, wovon gesprochen wird, auch im Körper gespürt wird und wenn für körperlich empfundene und emotional gespürte Phänomene die richtigen Worte gefunden werden, verdichten sich diese Erfahrungen zu stimmigen Einsichten, Einstellungen und Überzeugungen.

Menschen brauchen attraktive und sinnliche Erfahrungen von verändertem Verhalten, um neuronal stabil gebahnte und habituell verankerte hartnäckige Gewohnheiten verändern zu können. Für die Vorwegnahme, im Planen und Imaginieren, werden Handlungsoptionen und Entscheidungen als richtig erlebt, wenn sich zu der Erkenntnis und dem Vorhaben auch körperlich ein Gefühl von Stimmigkeit und Gewissheit einstellt. In einem gelungenen Entscheidungsprozess wird das „Wechselverhältnis zwischen einem expliziten, überlegten Sinn für das, was ich tun möchte, was möglich ist, und einem mehr intuitiven Spürsinn, einem impliziten Sinn für das, was für mich affektiv, leiblich stimmig erscheint", in einen Dialog gebracht (Fuchs und van Kaldenkerken 2018, S. 17). In Bezug auf die Vergangenheit lassen sich im nachfühlenden, spürenden Erinnern Szenen mit allen Sinneseindrücken wiederbeleben. Das kann für das Aufspüren von Triggern und Knotenpunkten in Mikroanalysen, aber mehr noch für die Belebung von Ressourcen und stärkenden Erfahrungen hilfreich sein.

Um als Berater/in mit nonverbalen, leiblichen und atmosphärischen Phänomenen in einer begleitenden, nicht deutenden Weise zu arbeiten, ist die Verbundenheit und die Vertrautheit mit sich selbst grundlegend für eine offene, freischwebende Aufmerksamkeit. Begriffe wie geteilte oder pendelnde Aufmerksamkeit sind andere Beschreibungen für die Fähigkeit, mit dem Anderen oder den Anderen, mit der Atmosphäre in der Gruppe mitzuschwingen, gleichzeitig mit sich verbunden zu sein, die eigenleiblichen Empfindungen, Resonanzen und begleitenden Gedanken wahr- 
zunehmen und zu beobachten. Es ist die Schwingungs- und Wahrnehmungsfähigkeit für den nichtsprachlichen Ausdruck, für die Wortwahl, Stimmmodulation u. a., ohne sich selbst zu verlieren und zu deuten. Dies auszubilden und zu üben, ist meines Erachtens eine wichtige und erlernbare Fähigkeit, die zur Grundausstattung und Ausbildung von Berater/innen gehört.

\section{Dritte Vertiefung: Die Verbindung von Körper/Leib und Geist/Psyche}

Wir bemerken sehr eindrücklich unsere Gefühle an Veränderungen in der gesamten Körperlandschaft, wie es in vielen Redewendungen zum Ausdruck kommt: Da schlägt einem das Herz im Hals, es wird mir eng ums Herz, es schnürt einem den Atem zu und man bekommt weiche Knie. Am eigenen Leibe spüren wir deutlich die Verbindungen und Wechselwirkungen von Körper/Leib, Geist und Umwelt, wenn der Körper mit starken positiven oder negativen Gefühlen auf äußere Eindrücke reagiert oder mit Krankheit oder basalen Bedürfnissen aus dem latenten Hintergrunderleben heraus in die Wahrnehmung tritt.

Jeder Eindruck aus der äußeren Welt, jede wiederbelebte Erinnerung und Erfahrung lösen komplexe körperlich-emotionale Reaktionen aus, an denen das zentrale und autonome Nervensystem, Herz und Kreislauf, Muskulatur und Atmung, innere Organe beteiligt sind. In diesem zirkulären Wechselspiel von biologischen, emotionalen, gedanklichen, motorischen Vorgängen lassen sich kognitive, psychische Vorgänge nicht isoliert betrachten. Viele Experimente und Forschungsergebnisse haben den wechselseitigen Einfluss verschiedener Körpervariablen (Körperhaltung, Mimik, Gestik u. a.) auf Emotionen, motivationale Prozesse, neurobiologische Vorgänge, Wahrnehmungen, Einstellungen und Bewertungen aufgezeigt. In den Zusammenfassungen zentraler Forschungsergebnisse (Storch et al. 2018) wird nachvollziehbar, wie Emotionen, motivationale Prozesse, Gehirnaktivitäten und Wahrnehmungen, Einstellungen und Bewertung untrennbar und unbewusst von mimischen und gestischen Ausdrucksbewegungen und Körperhaltungen beeinflusst werden.

Interessant für die Beratung ist, dass die Interventionen in den zirkulären Beeinflussungen von Emotionen, Bewertungen, körperlichen Vorgängen und begleitenden, unterschwelligen körperlichen Bewegungen an ,,jeder Stelle“ gesetzt und durch die fortlaufenden zirkulären Prozesse weitergetragen werden. So wie die Änderung von Einstellungen sich in einer veränderten Körperhaltung zeigen wird, ist es möglich, über eine veränderte Haltung und Bewegung Einstellungen zu verändern. Das bedeutet allerdings nicht, dass wir allein mit ,positiven“ Posen oder Ausdrucksbewegungen unsere Emotionen und Bewertungen direkt beeinflussen können, wie es z. B. Cuddy (2020) vorschlägt. ,Folgt man jedoch den Argumenten von Barsalou (2009, 2015), dann ist ein solches pauschal imitierendes Vorgehen nicht sinnvoll, denn die Bedeutung einer Pose wird erzeugt durch die individuelle Erfahrung und die daran gekoppelte Simulation von Körperverfassungen, ist also immer abhängig von dem, was ein Individuum an Werten, kulturellen Normen und persönlichen Erinnerungen gespeichert hat" (Storch et al. 2018, S. 20).

Eine Haltung sollte und kann man nicht direktiv anleiten, so wie alle Interventionen immer nur Anregungen sein können. Nicht mit Instruktionen und Kontrolle von 
außen, sondern über eine spezifische Form der Aufmerksamkeit, Selbsterkundung und Selbstwahrnehmung können wir in der Beratung mit den Elementen Haltung, Bewegung, Stimme, Atmung, Stand auf experimentelle Weise probehandelnd arbeiten und Veränderungen von Einstellungen und Gefühlen anregen. Die verkörperte Haltung als Anker für den Transfer in die Praxis beachtet die Rolle des Körpergedächtnisses für Lern- und Veränderungsprozesse.

\section{Vierte Vertiefung: Verkörperte Begegnung - Zwischenleibliche Resonanz}

Wenn zwei Personen angeregt und intensiv miteinander sprechen, kann man häufig Spiegelungen in den Gesten und Körperhaltungen beobachten. Sie neigen sich einander zu, überschlägt einer ein Bein, folgt die andere. Von Gesichtsmimikry ist die Rede, wenn sich das Lächeln wie automatisch im Gesicht des Gesprächspartners fortsetzt. Es wirkt wie ein gut abgestimmter Paartanz, indem mal der eine und mal die andere führt. Die unbemerkte präverbale Synchronie ist charakteristisch in dyadischen Interaktionen und beginnt, sobald Menschen miteinander in Kontakt treten. Ob die „Chemie“ stimmt, spüren wir an einer solchen Synchronie und einer leiblich empfundenen Stimmigkeit. Das gilt auch für die Herstellung einer guten Beratungsbeziehung.

Obwohl wir uns in der Beratung überwiegend auf die verbale, explizite Interaktion konzentrieren, sind wir auf einer unterschwelligen, präverbalen Ebene miteinander verbunden. Die unterschwellige Interaktion ist ein maßgeblicher Teil der Beratungsinteraktion, eine gemeinsam gestaltete Atmosphäre und tragende Verbundenheit, die sich im unbewussten Angleichen von Gesten, Mimik, Stimmmodulation, Körperhaltungen und sogar im Herzschlag zeigt. Vertrauen, Kontakt und Sicherheit als tragende Basis und wichtiger Wirkfaktor einer guten Arbeitsbeziehung werden nicht verbal versichert, sondern leiblich gespürt.

Das Resonanzgeschehen wirkt über die Verbindung zweier Kreisläufe von innerleiblicher und zwischenleiblicher Resonanz. In einer systemischen Interaktionsdynamik verknüpft sich das vertikale, innerleibliche Resonanzgeschehen horizontal auf der zwischenleiblichen Ebene mit dem des Gegenübers und bildet die Basis für leibliche Empathie und soziales Verstehen. Wie oben beschrieben, ist jeder Eindruck aus der äußeren Welt, jede wiederbelebte Erinnerung und Erfahrung mit körperlichemotionalen Reaktionen verbunden, die über die Qualitäten und Relevanzen der Eindrücke informieren. Die eigenleibliche Resonanz ist unmittelbar körperlich mit Bewegungsimpulsen verknüpft, die wiederum in rudimentären Handlungsbewegungen zum Ausdruck kommen. In der verkörperten sozialen Interaktion werden Mimik, Gestik, Haltung und die Andeutungen von Bewegungen wieder zum Eindruck für das Gegenüber und bilden so ein Wechselspiel von sich gegenseitig beeinflussenden Ein- und Ausdrücken. Merleau-Ponty (1966) nennt dieses spezifische Resonanzgeschehen des wechselseitigen körperlichen Verstehens ,Zwischenleiblichkeit“.

Forschungsergebnisse bestätigen den Zusammenhang von Synchronie und Beziehungsqualität. „Mehrere Analysen der Psychotherapieforschung weisen inzwischen jedoch darauf hin, dass sich in Bewegungs- und physiologischer Synchronie die 
Qualität der Therapiebeziehung spiegelt" (Tschacher 2019, S. 10). Positive Affektivität verkörpert sich in gemeinsamen Bewegungen. Synchronie in der dyadischen therapeutischen Interaktion steht im Zusammenhang mit einem positiven Therapieverlauf, Symptomreduktion, der Erreichung der individuellen Therapieziele, weniger interpersonaler Probleme und Selbstwirksamkeit. Durch das zwischenleibliche Geschehen ,wird ein Großteil der eigentlichen Wirksamkeit von Gesprächen und Psychotherapie transportiert" (Fuchs und van Kaldenkerken 2018, S. 14).

Die Fähigkeit, sich aufeinander einzustellen, einzuschwingen und intuitiv zu verstehen, mitzufühlen und in emotionale Resonanz und spontanes Verstehen mit anderen Menschen zu kommen, wird in den ersten Lebensjahren erlernt und als frühkindliches Beziehungswissen im Leibgedächtnis gespeichert. Menschliche Wahrnehmung ist mehr als eine subjektive Perspektive. ,Wir sehen, hören, tasten und behandeln die Dinge gewissermaßen immer auch mit den Augen, Ohren und Händen der anderen“ (Fuchs 2020, S. 149; vgl. Fuchs 2017). Auf einer präverbalen, unterschwelligen Ebene vollzieht der Leib Bewegung, Mimik und sogar Bewegungsabsichten mit und erahnt das mögliche weitere Geschehen.

Die Entdeckung des neuronalen Spiegelsystems (Rizzolatti und Sinigaglia 2014) lieferte eine Erklärung für diese Resonanz- und Früherkennungsvorgänge. Dabei handelt es sich nicht um eine Simulation und eine irrtumsfreie Wahrnehmung, so als würden sich die Bewegungsabsichten oder Gefühle einfach doppeln. Sensomotorische und sensorisch-emotionale Spiegelneurone aktivieren sich und sind besonders stark, wenn das wahrgenommene Geschehen persönlich bekannt, besonders vertraut und wichtig ist. Erst die eigenen Erfahrungen mit Bewegungsabsichten, sozialen Interaktionen und Gefühlen ermöglichen überhaupt das Nachempfinden und Nachvollziehen. Ist eine Bewegung oder ein Gefühl nicht bekannt, werden sich die Spiegelneurone nicht aktivieren. Mit der Aktivierung werden „nur“ die spezifischen Erfahrungen, die eigenen Bewegungsgewohnheiten und vertrauten Gefühle und Bedeutungen spürbar und wirken innerleiblich zurück. Die Resonanz ist nicht die Abbildung des leiblichen Erlebens des Gegenübers.

Auch wenn typische Verläufe anregender intersubjektiver Reflexion und gemeinsamer Sinnkonstitution in zirkulären, verbundenen, spiralförmigen Wechselprozessen verlaufen, sind diese synchronen Austauschsituationen nicht durchgängig in einem harmonischen Einklang. Kleine Brüche, Disharmonieren, Fehlpassungen und manchmal ein längerer Verlauf von Missverständnissen müssen geklärt, verstanden und angepasst werden. Das spiegelt sich auch in der zwischenleiblichen Synchronie und wird auch leiblich als Unstimmigkeit gespürt. In gelingender Kommunikation lassen sich Anpassungsbewegungen und Synchronisationsbemühungen auch auf der nonverbalen Ebene beobachten.

Von einem pragmatischen Wahrheitsbegriff (Joas et al. 2020) ausgehend, gibt es auch keine allgemeinen, vorgegebenen Kriterien, um die Bedeutung oder die Wahrheit von Aussagen oder Situationen zu bewerten. Wahrheit in sozialen Systemen ist das Ergebnis von kommunikativen Verständigungsprozessen und ein Ereignis. Eine leiblich gespürte, nonverbale Synchronie ist also nicht nur ein markanter Indikator für die Beziehungsqualität, sondern auch ein Marker für gemeinsame Stimmigkeit und Sinnbildung. 
Die Modellierungen exzellenter Psychotherapeuten (Perls, Satir und Erickson) durch die Begründer des Neurolinguistischen Programmierens (NLP) haben bereits vor mehr als 40 Jahren diese Synchroniephänomene exploriert und als „Gesprächstechnik" bekannt gemacht (Bandler und Grinder 1988). Mit dem körperlichen Spiegeln (Pacen und Leaden) wird der nonverbale Kontakt bewusst unterstützt. Über das feine Spiegeln der kleinen, latenten Begleitbewegungen, der Haltung, Atemtiefe u. a. lassen sich im Gespräch unbemerkt Kontakt anbieten, Sicherheit schaffen, emotionale Qualitäten erspüren und als Hypothesen nutzen.

Durch diese Interaktionsschleifen können Interventionen an jeder Stelle ansetzen und durch die zirkuläre Verbundenheit im Ganzen wirksam werden. Das Wissen über die eigen- und zwischenleibliche Verbundenheit und die ,gegenseitige emotionale Ansteckung“ erklärt uns die Eskalationsdynamiken, besonders in Konflikten und Krisen. Mit diesem Kenntnisstand empfiehlt es sich, bei schwieriger Stimmungslage nicht allzu sehr mitzuschwingen, sondern die „Ansteckung umzudrehen“ und damit Stabilität, Resilienz, Ruhe, Zuversicht und das Zutrauen der Berater/in wirken zu lassen.

Wie auch in der verbalen Kommunikation führt das pure und offensichtliche körperliche Spiegeln zu Irritationen. Wenn das absichtsvolle körperliche Spiegeln zur Technik wird und an Echtheit, Verbundenheit und wirklichem Interesse verliert, verstört das aufkommende Misstrauen die Arbeitsbeziehung. Für die videobasierte Beratung vermutet Tschacher (2019, S. 11) eine beschränkte Reichweite. „Wenn die nonverbale Synchronie fehlt, etwa beim Austausch in sozialen Medien und Internetforen, fehlt die Echtheit wirklicher Kommunikation“.

Die Einschränkungen, die wir aktuell mit den Corona-Hygienemaßnahmen erleben, ermöglichen die Aufmerksamkeit für Alternativen, die in unserer Kultur und Lebensweise nicht gewohnt, praktiziert und geübt sind. Wenden wir uns dem Blickkontakt zu: Was passiert, wenn sich die besondere Nähe und Zuneigung nicht in der Umarmung ausdrücken kann und durch den Blickkontakt ersetzt wird? Wir sind nicht sehr geübt, uns in einer Weise anzusehen, in einem Blickkontakt zu sein, der Verbindung schafft. In-Verbindung-Sein lässt sich über den Blick, die Erfahrung, gesehen zu werden, besonders intensiv herstellen (Storch und Tschacher 2014).

\section{Fünfte Vertiefung: Die zwischenleibliche Resonanz als ,Diagnose- Instrument" - eine grundlegende Orientierung}

Die Resonanz- und Schwingungsfähigkeit ist ein zentrales ,diagnostisches“ Sensorium für die Einschätzung von Reflexions- und Handlungsfähigkeit. Die Fähigkeit zur komplexen Reflexion ist unmittelbar mit der emotionalen Lage eines Systems verbunden, die sich in der zwischenleiblichen Resonanz vermittelt.

Die besondere Qualität von Supervision und Coaching liegt in der reflexiven, mehrdimensionalen Bearbeitung beruflicher Themen. Diese Qualität ist nur möglich, wenn sich das emotionale System in einer entspannten Mittellage befindet. Ein Kennzeichen hierfür ist die Fähigkeit, sich zu sich selbst ins Verhältnis zu stellen, wie im Absatz über die Körper-Leib-Unterscheidung beschrieben wurde. Es gibt viele Momente, in der diese emotionale Mittellage nicht mehr gegeben oder in 
den Beratungssituationen nicht selbstverständlich vorgefunden wird. Die folgenden Aspekte sollten eine Orientierung für die Auswahl von Beratungsformaten und die Indikation von nichtsprachlichen Interventionen bieten.

Beschleunigung (Rosa 2013), ein hoher Entscheidungsdruck bei knapper Zeit und steigender Verantwortung, Ungewissheit und Unsicherheit bilden eine Art Grundtonus in vielen Bereichen der Arbeitswelt und auch der Lebenswelt. Das führt zu einem Stresserleben, das physiologisch die Reflexionsfähigkeit einschränkt, die wir aber für die komplexe Nachdenklichkeit in den Beratungen brauchen.

1. In Konflikten verlieren die betroffenen Personen gelegentlich ihre Handlungsfähigkeit in einem Maße, dass sie nicht mehr klar denken, geschweige denn ihre Interessen artikulieren und vertreten können. Sie geraten in einen physiologischen „Alarmzustand“, in dem kein Repertoire mehr für soziales Verhalten zur Verfügung steht (Porges 2018). Die Person ist nicht mehr in der Lage, zu diesem Zustand in Distanz zu treten, geschweige denn das Geschehen und sich selbst reflexiv und kritisch zu betrachten.

2. Das intensiviert sich noch in hocheskalierten Konflikten, bei traumatisierenden Ereignissen und in schweren Krisen. Hier spricht Porges von einem physiologischen Zustand des shutdown, indem die Betroffenen funktional immun für soziale Interaktionen sind.

3. Art und Stärke der Belastung und die Unterscheidung der Ebenen Problem, Konflikt und Krise (van Kaldenkerken 2014) sollten Berater/innen einschätzen können. Die „Daten“ lassen sich schwer aus der sprachlichen Beschreibung explizieren, sondern erschließen sich überwiegend über leiblich-atmosphärische Merkmale und eine Veränderung der Sprachmuster.

In solchen Zuständen hilft Reden nicht, und es ist gut zu wissen, dass die betroffene Person überhaupt nicht mehr besonnen handeln kann, zu keinem Perspektivenwechsel in der Lage ist und die Emotionen häufig nicht gut allein regulieren kann. Für eine faire Konfliktbearbeitung z. B. in der Mediation (van Kaldenkerken 2019) müssen Settings, Angebote und ein methodisches Repertoire zur Verfügung stehen, um die Fähigkeit zur Selbstvertretung wiederherzustellen.

Neben reflexionsunterstützenden Verfahren brauchen Berater/innen ein methodisches Repertoire für den Umgang mit brisanten und heftigen Emotionen und Krisen. Körperliche Interventionen unterstützen dann buttom up wesentlich schneller und wirkungsvoller die Emotionsregulation und Wiederherstellung der Handlungsund Reflexionsfähigkeit als sprachlich-kognitive Zugänge. Es gibt eine Vielzahl an Möglichkeiten, die unmittelbare Impulskraft von starken Emotionen zu reduzieren. Einfache körperliche Interventionen, wie z.B. Zentrierungsübungen, die Ausrichtung auf die Atmung oder bifokale Techniken (bekannt aus der Traumatherapie), sind Elemente einer solchen ersten Hilfe für Krisen. Der Body Scan, beruhigende Atem- und Selbstberührungstechniken führen die Betroffenen auf ihre eigene leibliche Wahrnehmung, zentrieren und beruhigen. Hier sei ausdrücklich betont, dass Berührungen tabu sind und der Grundsatz guter Beratung gilt, dass jede Intervention nur mit Zustimmung der Beteiligten erfolgt. 


\section{Abschließende Überlegungen und Bewertungen}

Mit der Frage, in welcher Weise sich Körper und Geist mit der Umwelt verschränken und ob und wie diese Dimension Teil der Reflexion sein kann und sollte, schließt der Beitrag ab.

Die leiblich gespürte Verunsicherung durch gesellschaftliche Ereignisse, Sorgen wegen Corona, der amerikanischen Wahlen, Klimakrise u. a. verdichten sich über die Person hinaus zu Atmosphären. Atmosphäre lässt sich als übergreifendes räumliches Geschehen, als psychische Verfassung einer Gruppe oder einer Gesellschaft verstehen, die nicht mehr einem einzelnen Subjekt zuzuschreiben ist, jedoch subjektiv erlebt wird und als atmosphärische Umgebung wieder zurückwirkt.

Auch wenn sich der Anteil an Fachberatung, Reflexion und Handlungsvorbereitung in den Formaten Supervision, Coaching und Organisationsberatung unterschiedlich gewichtet, ist Reflexion für alle Formate ein wichtiger Modus. Im Format Supervision wird Reflexion als zentrales Merkmal beschrieben. Die Orientierung an humanistischen Werten und am Gemeinwohl gelten als leitende ethische Werte für das Beratungshandeln und das kollegiale Miteinander im Berufsverband DGSv (ethische Leitlinien der DGSv). Dieser Anspruch gerät zunehmend in Gefahr. Die dominante Zeitstruktur der Gegenwart und das damit verbundene Lebensgefühl vieler Menschen in westlich geprägten Gesellschaften lassen sich mit dem Begriff der Beschleunigung (Rosa) auf den Punkt bringen. Die inhärente Steigerungsdynamik des Beschleunigungsprozess hat das Potenzial, ,soziale Störungen zu erzeugen, die menschliches Leiden und Unzufriedenheit hervorrufen“ (Rosa 2013, S. 66).

In westlichen Zivilisationen ist die historisch und kulturell gewachsene Vorstellung von Zeit als linear ausgerichteter, kontinuierlicher, fortschreitender Prozess selbstverständlich. Diese tendenziell ungebremsten linearen Prozesse geraten in eine Beschleunigungsdynamik, die kaum Hemmung erfahren und in Beratungen immer häufiger thematisiert werden. Wir erschaffen uns eine Lebens- und Arbeitsumgebung, die nicht nur unsere Lebensgrundlagen ausbeuten und zerstören, sondern unseren seelischen und körperlichen Rhythmen und der Notwendigkeit zur Regeneration und Gesundheit zuwiderlaufen und uns überfordern. Im linearen Zeitverständnis „gibt es kein Ziel, dem die Zeit zustrebt, an dem man zur Ruhe kommt. Alles bewegt sich vorwärts, so auch unser Leben. Wir laufen immer weiter voran, kommen aber nicht wirklich an“ (Fuchs 2019, S. 39f.). Dies führt zu der Frage, wie lange sich eigenleibliche Rhythmen ignorieren, anpassen und optimieren lassen.

Die Zeitlichkeit des Lebendigen dagegen ist eine zyklische Zeit, die immer wieder in der Gegenwart ankommt. Die regelmäßig wiederkehrenden Prozesse von Aktivität und Entspannung, Wachheit und Schlaf, Essen und soziale Zuwendung geraten in Widerspruch zu der linearen Dynamik in einem wachstumsorientierten Wirtschaftssystem, wenn die zyklischen Prozesse missachtet werden. Unser Leib ist konservativ und ,antiquiert“ (Anders 2018 [1956]), und die Eigenzeiten des Leibes können nicht ohne Schaden beschleunigt, kompensiert und optimiert werden. „Zyklische und lineare Zeit stehen in Spannung zueinander, und vielfältige kollektive und individuelle Pathologien gehen auf eine Entkopplung der linearen Dynamik von den Lebensprozessen zurück, insbesondere auf eine Beschleunigung und Entrhythmisierung der sozialen und individuellen Zeitabläufe“" (Fuchs 2020, S. 315). 
Die Bedingungen des Lebendigen, die Rahmenbedingungen der menschlichen Existenz setzen der ungebremsten Dynamik deutliche Grenzen. Immer häufiger sind die Menschen und zu beratenden Systeme gar nicht in der Verfassung, komplex nachzudenken. Das betrifft besonders Führungs- und Fachkräfte mit viel Verantwortung und hoher Entscheidungsdichte. Der Arbeitsdruck und die beruflichen Anforderungen führen zu einem Stresserleben, das physiologisch mit einer eingeschränkten Reflexions- und Denkfähigkeit und mit abnehmender Selbstbestimmung im Sinne der exzentrischen Positionalität (Plessner 1982) einhergeht.

Supervision, Coaching und Organisationsberatung konzipieren sich mittlerweile zwischen den Polen von Anpassung und Gestaltung. Wenn die Funktionalität sehr im Vordergrund steht und die leitenden Vorannahmen dieser Dynamik nicht mitreflektiert werden, kann Beratung nur Anpassungsleistungen vollbringen. Die Überforderung der Subjekte wird dann individualisiert, Gesundheit wird zur persönlichen Angelegenheit, und Beratung wird der Ort, an dem Resilienz gefördert, Ressourcen entwickelt und Überlastungssymptomen vorgebeugt werden soll. Für Berater/innen stellt sich dann die Frage, ob sie ihre Beratungen an die skizzierten Rahmenbedingungen anpassen, die VUKA-Welt als nicht zu ändernde Umwelt akzeptieren und in das Selbstverständnis und Tempo zustimmend einsteigen.

Berater/innen sind gut vertraut mit der individuellen und sozialen Reflexion von parafunktionalen Glaubenssätzen und Vorannahmen von Einzelnen und Gruppen und kennen die handlungsleitende Kraft von Glaubenssätzen und Überzeugungen aus der täglichen Praxis. Was wir als einzelne Berater/innen, als Beratungsfirmen, Professionsgemeinschaften und Berufsverbände seltener in den Blick nehmen, sind die leitenden ökonomischen und politischen Glaubenssätze und Überzeugungen, die latent und unhinterfragt in die Beratung (und die Gesellschaft) wirken. Wenn VUKA oder Arbeitswelt 4.0 als unveränderliche Rahmenbedingungen der arbeitsweltlichen Beratung von vielen Berater/innen akzeptiert und integriert werden, stellt sich die Frage, warum dieses Wettbewerbsparadigma höher bewertet wird als Gesundheit und Nachhaltigkeit. Die Alternative dazu sehe ich in der Orientierung an den UNNachhaltigkeitszielen 2030, den WHO-Salutogenese-Kriterien für gute Arbeit oder in der Gemeinwohlverpflichtung als DGSv-Mitglied.

Wenn die leiblichen Ressourcen so eindeutige Hinweise geben und die Eigenzeiten des Lebendigen als Gegenprinzip der linearen Dynamik Grenzen setzen, dann sollten diese Widersprüche als Kernkonflikt (Schwarz 1990) guter Arbeit ein Teil der Reflexion sein, die über die Auftrags- und Auswertungsgespräche mit den Vorgesetzten, OE- und PE-Abteilungen in die Organisation verbunden werden. Reflexive Beratungen sind zirkulär angelegt und folgen idealerweise zyklischen Rhythmen. Es sind Besinnungs-, Begegnungs- und Gegenwartsorte, die sich von den linearen, beschleunigten Prozessen absetzen. Im Verständnis einer verkörperten Perspektive von zirkulären wechselseitigen Beeinflussungen durch und zur Umwelt wird das Ausloten der Handlungsmöglichkeiten und der Einflussnahme Teil der Beratung. Eine ,abstinente“ Haltung ist dann allerdings schwer durchzuhalten, wenn man eine „,parteiliche“ Nähe für die Qualität, die Aufgabe der Organisation, den Organisationszweck, das Gemeinwohl und die Gesundheit von Mitarbeiter/innen einnimmt. „Die Qualität der Konvivialität umfasst Verbundenheit in einer Leichtigkeit des Miteinanderseins, wo jeder so sein kann und akzeptiert wird, wie er ist, und so eine 
,Konvivialität der Verschiedenheit" möglich wird, wo ein Raum der Sicherheit und Vertrautheit gegeben ist, eine gewisse Intimität integrer Zwischenleiblichkeit, in der man ohne Furcht vor Bedrohung, Beschämung, Beschädigung, ohne Intimidierung zusammen sitzen, beieinander sein kann, weil die Andersheit unter dem Schutz der von allen gewünschten, gewollten und gewahrten Gerechtigkeit steht, und jeder in Freiheit (parrhesiastisch) sagen kann, was er für wahr und richtig hält" (Petzold 2007, S. 399).

Eine solche Vorstellung von Respekt, Dialog und „,moralischer Intimität“, wie Bieri (2013) diese feine soziale Achtsamkeit nennt, die die Bedürfnisse der anderen in die Aufmerksamkeit und das Handeln mit einbezieht, sollte das Leitbild für Reflexion bleiben, auch wenn es sich selten in dem oben beschriebenen Maße herstellen lässt. Je mehr sich von dieser Atmosphäre und integrer Zwischenleiblichkeit einstellen kann, desto komplexer und anspruchsvoller kann berufliche Reflexion werden. Es lohnt sich, Zeit und Rahmenbedingungen für solche Reflexionsräume zu schaffen, um schnelle Lösungen für komplexe Probleme, zu schnelles gewohnheitsmäßiges Handeln, das Ignorieren eigener somatischer Marker bei unstimmigen Entscheidungen zu vermeiden.

Die Beschäftigung mit der Verkörperungsperspektive macht deutlich, dass die vermeintlichen Polaritäten von Körperhaben und Leibsein, von Anpassung und Gestaltung, von Ergebenheit und Selbstbestimmung, Technologieentwicklung und -kritik und von linearer Beschleunigung und Verlangsamung keine unauflösbaren Widersprüche sind. Es geht um verschiedene Rhythmen, Logiken und Eigenzeiten, die dem technologischen Fortschritt, unserem Wirtschaftssystem und dem lebendigen Leben innewohnen, und um eine Balance in dieser Dialektik. Die meisten Veröffentlichungen fokussieren die Körper-Geist-Verbindung und die Kopplung zur Umwelt in Bezug zur intersubjektiven und räumlichen Dimension. Die Verkörperungsperspektive legt die gleichwertige Betrachtung der wechselseitigen Beeinflussungen auch mit gesellschaftlichen Atmosphären nahe.

\section{Literatur}

Anders, G. (2018). Die Antiquiertheit des Menschen 1. Über die Seele im Zeitalter der zweiten industriellen Revolution. München: C.H. Beck.

Antonovsky, A. (1997). Salutogenese. Zur Entmystifizierung der Gesundheit. Tübingen: DGVT.

Bachmann, T. (2020). Die Kontaktgrenze in dynamischen Zeiten oder Gedanken zum Coaching 4.0. Organisationsberatung, Supervision, Coaching, 27(3), 287-298.

Bandler, R., \& Grinder, J. (1988). Neue Wege der Kurzzeit-Therapie. Neurolinguistische Programme. Paderborn: Junfermann.

Barsalou, L. W. (2009). Simulation, situated conceptualization and prediction. Philosophical Transactions of der Royal Society, 364, 1281-1289.

Barsalou, L. W. (2015). Situated conceptualization: theory an application. In Y. Coello \& H. M. Fischer (Hrsg.), Foundation of embodied cognition. East Sussex: Psychology Press.

Bieri, P. (2013). Wie wollen wir leben? München: Dtv.

Böhme, G. (2017). Leibsein als Aufgabe. Leibphilosophie in pragmatischer Hinsicht. Zug: Die graue Edition.

Cuddy, A. (2020). Power posing. https://de.wikipedia.org/wiki/Power_Posing. Zugegriffen: 19. Okt. 2020.

Damasio, A.R. (2004). Descartes Irrtum. Fühlen, Denken und das menschliche Gehirn. Berlin: List/ Ullstein.

Damasio, A. R. (2011). Ich fühle, also bin ich. Die Entschlüsselung des Bewusstseins. Berlin: List, Ullstein. 
Fuchs, T. (2017). Das Gehirn - ein Beziehungsorgan. Eine phänomenologisch-ökologische Konzeption (5. Aufl.). Stuttgart: Kohlhammer.

Fuchs, T. (2019). Schneller, als die Zeit erlaubt. Psychologie heute, 10, 39-42.

Fuchs, T. (2020). Verteidigung des Menschen. Grundlagen einer verkörperten Anthropologie. Berlin: Suhrkamp.

Fuchs, T., \& van Kaldenkerken, C. (2018). Interview: Verkörpertes Gedächtnis, zwischenleibliche Interaktion und nachhaltige Veränderungen. supervision Mensch - Arbeit-Organisation, 36(3), 12-18.

Gallagher, S. (2012). Kognitionswissenschaften - Leiblichkeit und Embodiment. In E. Alloa, T. Bedorf, C. Grüny \& T. N. Klass (Hrsg.), Leiblichkeit (S. 320-333). Tübingen: Mohr Siebeck.

Gugutzer, R. (2015). Soziologie des Körpers. Bielefeld: transcript.

Joas, H., Schubert, J.H., \& Wenzel, H. (2020). Pragmatismus zur Einführung. Hamburg: Junius.

van Kaldenkerken, C. (2014). Wissen was wirkt. Modelle und Praxis pragmatisch-systemischer Supervision. Hamburg: Tredition.

van Kaldenkerken, C. (2019). Selbstverantwortung stärken. Selbstklärung mit PEP in der Mediation. In M. Bohne \& A. Ebersberger (Hrsg.), Synergien nutzen mit PEP. Die integrative Kompetenz der Prozess- und Embodiment-fokussierten Psychologie in Psychotherapie, Beratung und Coaching (S. 244-253). Heidelberg: Carl-Auer.

Merleau-Ponty, M. (1966). Phänomenologie der Wahrnehmung. Berlin: De Gruyter.

Milz, H. (2019). Der eigensinnige Mensch. Körper, Leib \& Seele im Wandel. Aarau, München: AT Verlag.

Papst Franziskus (2020). Fratelli tutti. https://www.katholisch.de/artike1/27094-fratelli-tutti-papstfranziskus-programm-fuer-eine-bruederliche-welt. Zugegriffen: 19. Okt. 2020.

Petzold, H. G. (2007). Integrative Supervision, Meta-Consulting, Organisationsentwicklung. Wiesbaden: VS.

Petzold, H. G. (2016). POLYLOGE 30/2016. http://www.fpi-publikation.de. Zugegriffen: 11. Okt. 2020.

Plessner, H. (1982). Mit anderen Augen. Aspekte einer philosophischen Anthropologie. Stuttgart: Reclam.

Porges, S. (2018). Die Polyvagal-Theorie und die Suche nach Sicherheit. Lichtenau/Westfalen: G.P. Probst.

Rizzolatti, G., \& Sinigaglia, C. (2014). Empathie und Spiegelneurone: die biologische Basis des Mitgefühls. Berlin: Suhrkamp.

Rosa, H. (2013). Beschleunigung und Entfremdung. Berlin: Suhrkamp.

Schindler, S. (2020). Ein achtsamer Blick auf den Achtsamkeits-Hype. Organisationsberatung, Supervision, Coaching, 27(1), 111-124.

Schürmann, V. (2012). Max Scheler und Helmuth Plessner - Leiblichkeit in der Philosophischen Anthropologie. In E. Alloa, T. Bedorf, C. Grüny \& T. N. Klass (Hrsg.), Leiblichkeit (S. 207-223). Tübingen: Mohr Siebeck.

Schwarz, G. (1990). Konfliktmanagement. Sechs Grundmodelle der Konfliktlösung. Wiesbaden: Gabler.

Sternagel, J. (2012). Bernhard Waldenfels - Responsivität des Leibes. In E. Alloa, T. Bedorf, C. Grüny \& T. N. Klass (Hrsg.), Leiblichkeit (S. 116-129). Tübingen: Mohr Siebeck.

Storch, M., \& Tschacher, W. (2014). Embodied Communication. Kommunikation beginnt im Körper, nicht im Kopf. Bern: Huber.

Storch, M., Tschacher, W., \& Weber, J. (2018). Embodiment im coaching und training. Zeitschrift Supervision. Mensch Arbeit Organisation, 36(3), 19-24.

Tschacher, W. (2019). Wie synchron sind wir? TPS - Theorie und Praxis der Sozialpädagogik, 6, 8-11.

Waldenfels, B. (2000). Das leibliche Selbst. Vorlesungen zur Phänomenologie des Leibes. Frankfurt/M.: Suhrkamp. 


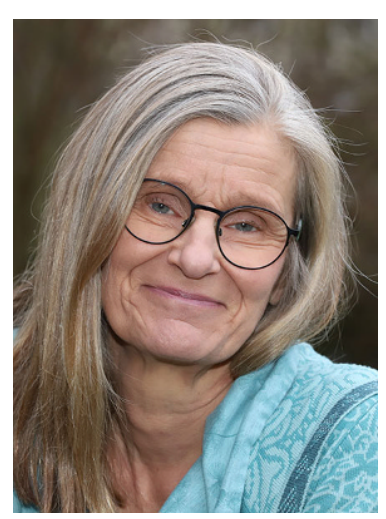

Carla van Kaldenkerken Dipl.-Sozialpädagogin, Seit 1988 Supervisorin und Coach DGSv, Mediatorin und Ausbilderin für Mediation $\left(\mathrm{BM}^{\circledR}\right)$, Organisationsberaterin, Geschäftsführende Gesellschafterin step GbR. Zuvor Tätigkeit in einer Unternehmensberatung für soziale Organisationen. Zusätzliche Qualifikationen als Bewegungs- und Tanzpädagogin, Feldenkrais, Psychodrama, NLP, Organisationaufstellung, $\mathrm{PEP}^{\circledR}$ und diversen körperintegrativen und -orientierten Methoden. Internet: www.step-beratung.de. 